\title{
High ionic strength narrows the population of sites participating in protein ion-exchange adsorption: A single-molecule study
}

\author{
Lydia Kisley $^{\mathrm{a}}$, Jixin Chen ${ }^{\mathrm{a}}$, Andrea P. Mansura, Sergio Dominguez-Medina ${ }^{\mathrm{a}}$, Eliona Kulla ${ }^{\mathrm{a}}$, \\ Marci Kang ${ }^{\mathrm{a}}$, Bo Shuang ${ }^{\mathrm{a}}$, Katerina Kourentzi ${ }^{\mathrm{b}}$, Mohan-Vivekanandan Poongavanam ${ }^{\mathrm{C}}$,

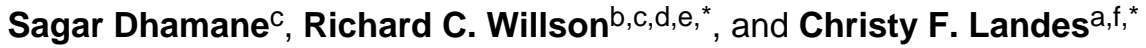 \\ a Department of Chemistry, Rice University, Houston, TX 77251 \\ b Department of Chemical \& Biomolecular Engineering, University of Houston, Houston, TX \\ 77004 \\ c Department of Biology \& Biochemistry, University of Houston, Houston, TX 77004 \\ d Houston Methodist Research Institute, Houston, TX, 77030 \\ e Centro de Biotecnología FEMSA, Departamento de Biotecnología e Ingeniería de Alimentos, \\ Tecnológico de Monterrey, Monterrey, NL 64849, Mexico \\ f Department of Electrical and Computer Engineering, Rice University, Houston, TX 77251
}

\begin{abstract}
The retention and elution of proteins in ion-exchange chromatography is routinely controlled by adjusting the mobile phase salt concentration. It has repeatedly been observed, as judged from adsorption isotherms, that the apparent heterogeneity of adsorption is lower at more-eluting, higher ionic strength. Here, we present an investigation into the mechanism of this phenomenon using a single-molecule, super-resolution imaging technique called motion-blur $\underline{\text { Points }}$ Accumulation for Imaging in Nanoscale Topography (mbPAINT). We observed that the number of functional adsorption sites was smaller at high ionic strength and that these sites had reduced desorption kinetic heterogeneity, and thus narrower predicted elution profiles, for the anionexchange adsorption of a-lactalbumin on an agarose-supported, clustered-charge ligand stationary phase. Explanations for the narrowing of the functional population such as inter-protein
\end{abstract}

(c) 2014 Elsevier B.V. All rights reserved.

*Corresponding Authors Dr. Christy F. Landes; Rice University; Department of Chemistry; P.O. Box 1892, MS 60; Houston, TX 77251; (713) 348-4232; cflandes@ rice.edu. * Dr. Richard C. Willson, University of Houston, Department of Chemical and Biomolecular Engineering, 4800 Calhoun Rd., Houston, TX 77004; (713) 743-4308; willson@uh.edu.

Lydia Kisley: 1k12@rice.edu

Jixin Chen: Jixin.chen@ rice.edu

Andrea P. Mansur: andrea.p.mansur@ rice.edu

Sergio Dominguez-Medina: Sergio.Dominguez-Medina@ rice.edu

Eliona Kulla: Eliona.Kulla@rice.edu

Marci Kang: Marci.Kang@rice.edu Bo Shuang: bs16@rice.edu

Katerina Kourentzi: edkourentzi@uh.edu

Mohan-Vivekanandan Poongavanam: mohan.vivekanandha@gmail.com

Sagar Dhamane: dhamane.edu@gmail.com

Publisher's Disclaimer: This is a PDF file of an unedited manuscript that has been accepted for publication. As a service to our customers we are providing this early version of the manuscript. The manuscript will undergo copyediting, typesetting, and review of the resulting proof before it is published in its final citable form. Please note that during the production process errors may be discovered which could affect the content, and all legal disclaimers that apply to the journal pertain. 
interactions and protein or support structural changes were investigated through kinetic analysis, circular dichroism spectroscopy, and microscopy of agarose microbeads, respectively. The results suggest the reduction of heterogeneity is due to both electrostatic screening between the protein and ligand and tuning the steric availability within the agarose support. Overall, we have shown that single molecule spectroscopy can aid in understanding the influence of ionic strength on the population of functional adsorbent sites participating in the ion-exchange chromatographic separation of proteins.

\section{Keywords}

mbPAINT; bioseparations; ion-exchange; heterogeneity; optical nanoscopy

\section{Introduction}

Ion-exchange chromatography is an essential tool of the modern pharmaceutical industry. The cost of purification of pharmaceutical proteins remains high and may reach up to 50\% of the total cost of goods [1]. This expense motivates efforts toward a more fundamental understanding of the complex competitive interactions of mixtures of proteins with heterogeneous polymeric adsorbents [2-4]. Adsorption isotherm measurements have traditionally been used to quantify the relationship between adsorbent properties, such as the dissociation constant and capacity, under varying ionic strength, $\mathrm{pH}$, and other mobile phase conditions. Adsorption data often are fit with empirically-determined constants that modify the Langmuir and other adsorption isotherm models [5-15]. Alternatives to the idealized Langmuir model have been applied to protein adsorption data, both to capture the expected non-idealities of the process, and to better fit the observed heterogeneities of protein adsorption data $[10,13,14]$.

A variety of sources can lead to the observed heterogeneity of protein adsorption, including intrinsic variance of the adsorbate molecule population due to differences in posttranslational modifications, translational inaccuracy, and post-synthesis modifications such as deamidation and oxidation $[6,16,17]$, inter-protein electrostatic repulsion and steric overlap [6,8], and irregularities in the adsorbent surface [18,19]. Because adsorption heterogeneity is observed even with uniform adsorbate populations and at very low loadings [20], it is likely that inherent heterogeneity of the adsorbent surface commonly plays the larger role. Adsorbent heterogeneity can arise from a range of potential causes, including steric accessibility and constraint, surface entropy and mobility [13], and the stochastic clustering of ligands on the adsorbent surface [21]. We have found that while adsorbents based on pre-organized, penta-valent clustered-charge ligands show higher protein affinity and capacity than adsorbents of the same total density of charge randomly distributed, isotherms for protein adsorption even on these nominally-homogeneous adsorbents show heterogeneity [12]. This observation further implicates adsorbent steric and surface mobility properties as a ubiquitous source of heterogeneity; especially as our recent single-molecule observations highlighted the role of both ligand clustering and steric availability with the porous support [21]. 
Adsorption in ion-exchange chromatography is the result of interacting influences of adsorbent heterogeneity and mobile phase composition. Ionic strength and (less frequently) $\mathrm{pH}$ often are used to tune adsorption characteristics [22], as they can influence the retention, resolution, and recovery of biomolecules [5-15] and can often provide non-denaturing elution. As discussed below, the observed heterogeneity often is lower at less-adsorptive conditions of higher ionic strength, as inferred from adsorption isotherm fit parameters $[12,13]$. Despite extensive and careful study, only limited mechanistic explanations of adsorption heterogeneity and its modulation by ionic strength are available. Studies using scanning confocal microscopy have shown that ionic strength influences pore diffusivity and capacity within porous agarose-based stationary phases [23,24]. Extension of confocal microscopy to include Raman vibrational spectroscopy has shown that over a range of solution conductivities, sorption can occur with only minimal changes in protein structure [25]. While these studies have offered insights into the role of ionic strength, ensemble averaging complicates approaches to an a priori understanding of ion-exchange chromatography [24]. Experiments accessing the molecular scale thus potentially could support a more fundamental understanding of mobile phase selection in biomolecular separations.

Single molecule spectroscopy avoids ensemble averaging to access the underlying molecular processes controlling protein-stationary phase interactions in separations. Foundational single molecule work resolved variability present at heterogeneous ion-exchange and reverse-phase stationary phase interfaces using confocal microscopy combined with fluorescence correlation spectroscopy and blip analysis [26-32] and total internal reflection microscopy [33-37]. We have recently extended single molecule techniques to the superresolution level ( $<250 \mathrm{~nm}$, below the diffraction limit of light) to investigate adsorptive separations at the single ligand-single protein level [21]. Super-resolution imaging allows for long-sought direct observations at the nanometer scale of the interfacial interactions that control chromatography. Using improved experimental and analysis methods, we showed the importance of charge-clustering of ligands and the kinetic heterogeneity even of nominally chemically-identical adsorption sites $[12,21,26,38]$. These results demonstrate the potential of single molecule techniques to advance a mechanistic understanding of chromatography difficult to obtain with ensemble techniques.

In this work, we apply single molecule super-resolution imaging to investigate the influence of ionic strength on the heterogeneity of protein ion-exchange functional adsorption sites, and therefore the heterogeneity of elution profiles. The system studied was a-lactalbumin on an agarose-supported uniformly clustered-charge ligand (penta-argininamide) stationary phase which we recently reported has higher protein capacity and affinity than dispersedcharge adsorbents of identical overall charge density [12,21,26,38]. We show that at high ionic strength, adsorption on lower affinity sites is suppressed, and heterogeneity is reduced, leaving a more uniform population of sites to participate. Circular dichroism spectroscopy shows that tuning the ionic strength does not significantly change the structure of $a$ lactalbumin, while optical imaging of model agarose microbeads shows a change in support structure. With the addition of single protein adsorption kinetics, our findings support the conclusion that a combination of electrostatic screening between the protein and ligand and 
the tuning of the steric availability within the agarose support leads to the reduction in heterogeneity. Overall, single molecule spectroscopy offers the potential for a more detailed mechanistic understanding of the influence of ionic strength on the ion-exchange separation of proteins.

\section{Experimental Methods}

\subsection{Sample preparation}

All materials were obtained from Sigma-Aldrich (St. Louis, MO) unless otherwise noted. Glass coverslips $(22 \times 22 \mathrm{~mm}$, No. 1; VWR, Houston, TX) were cleaned for $90 \mathrm{~s}$ in a basic TL1 solution (4\% (v/v) $\mathrm{H}_{2} \mathrm{O}_{2}$ [Fisher Scientific, Radnor, $\mathrm{PA}$ ] and $13 \%(\mathrm{v} / \mathrm{v}) \mathrm{NH}_{4} \mathrm{OH}$ ) at 80 ${ }^{\circ} \mathrm{C}$ and then with $\mathrm{O}_{2}$ plasma for 2 min at medium power (PDC-32G; Harrick Plasma, Ithaca, NY). Silicon templates (Grace BioLabs, Bend, OR) were placed on top of the cover slips, and a precision coat-bake system (200CBX; Brewer Science Cee, Rolla, MO) was used at 2000-3000 rpm to spin coat $1 \mathrm{~mL}$ of hot $1 \%$ (w/w) agarose solution (US Biological, Swampscott, MA) to prepare agarose thin films. Intra and inter-sample agarose film uniformity was investigated by spinning agarose on (opaque) silicon wafers and measuring film thickness by ellipsometry (J.A. Woolam M-2000 spectroscopic ellipsometer); agarose preparation and spinning conditions on silicon were identical to those used for deposition on (transparent) glass, on which ellipsometry was less reliable. Average film thickness of the film was estimated to be $80 \mathrm{~nm}$ using a Cauchy dispersion model. Film thickness was not detectably altered by rate of pouring of molten agarose, or by pre-cooling for $10 \mathrm{~min}$ before spin-coating (the agarose solution was spin-coated within 2 min of preparation). A custom flow chamber ( $1 \mathrm{~mm}$ height, with an elliptical opening of $12 \times 5 \mathrm{~mm}$; part no. $43018 \mathrm{C}$, Grace BioLabs) was then placed over the agarose thin film.

Charged penta-argininamide ligands were immobilized on the agarose surfaces using aldehyde functionalities. As described by Afanassiev et al. [39] the agarose hydroxyl groups were first activated by $30 \mathrm{~min}$ treatment with $20 \mathrm{mM} \mathrm{NaIO}_{4}$. The activated surfaces were washed with deionized water and dried of standing droplets under a stream of helium gas. A $43 \mu \mathrm{M}$ solution of the penta-argininamide peptide $\left(\mathrm{NH}_{2}\right.$-GGRRRRRamide, Biomatik, Wilmington, DE) in coupling buffer $\left(100 \mathrm{mM} \mathrm{Na} 2 \mathrm{HPO}_{4} / \mathrm{NaH}_{2} \mathrm{PO}_{4}\right.$ [EM Science, Gibbstown, NJ] and $150 \mathrm{mM} \mathrm{NaCl}$ [Mallinckrodt Chemical, St. Louis, MO], pH 7.2) was introduced to the activated surface and was coupled to the surface with several drops of 20 $\mathrm{mM} \mathrm{CNBH}_{4}$ (Pierce, Rockford, IL) at $4{ }^{\circ} \mathrm{C}$ for $30 \mathrm{~min}$. The sample was then rinsed with coupling buffer to remove excess uncoupled peptide. Unreacted aldehyde sites were reduced with $66 \mathrm{mM} \mathrm{NaBH}_{4}$ (in $25 \% \mathrm{EtOH} / 75 \%$ phosphate-buffered saline) at $4{ }^{\circ} \mathrm{C}$ for $5 \mathrm{~min}$. The final surfaces were rinsed with deionized water and stored at $4{ }^{\circ} \mathrm{C}$. The guanidinium side group of arginine $\left(\mathrm{pK}_{\mathrm{a}}=12.5\right)$ is protonated at $\mathrm{pH} 7.2$ and thus unreactive towards the agarose aldehyde groups, and coupling through the $\mathrm{N}$-terminal primary amine is strongly favored.

$\mathrm{Ca}^{2+}$-depleted a-lactalbumin (L6010) was prepared from unpasteurized bovine milk and was purified by ion-exchange chromatography on DEAE-agarose. The purity was $\geq 85 \%$ as determined by PAGE. The molar mass of the protein used was $14,100 \mathrm{~g} / \mathrm{mol}$ and the extinction coefficient $\varepsilon^{1 \%}=20.1$. a-Lactalbumin was labeled with Alexa 555 succinimidyl 
ester (Life Technologies; abs/em maxima: 555/565 nm). The fluorophore was dissolved to $10 \mathrm{mg} / \mathrm{mL}$ in dimethylformamide and $0.1 \mathrm{~mL}$ of the fluorophore solution was slowly added to $1 \mathrm{~mL}$ of $10 \mathrm{mg} / \mathrm{mL} \mathrm{Ca}^{2+}$-depleted a-lactalbumin dissolved in $0.1 \mathrm{M} \mathrm{NaHCO}_{3}$ buffer, $\mathrm{pH}$ 7.4 to preferentially label the amino terminus of the protein [40]. The reaction was incubated at room temperature for $1 \mathrm{~h}$ with constant gentle mixing. Freshly prepared hydroxylamine $(0.1 \mathrm{~mL}, 1.5 \mathrm{M}, \mathrm{pH} 8.5)$ was added to stop the reaction. The labeled protein was dialyzed for $24 \mathrm{~h}$ first against $\mathrm{H}_{2} \mathrm{O}$ and then against $10 \mathrm{mM}$ Tris, $100 \mathrm{mM} \mathrm{NaCl}$, pH 8.0. Gel filtration chromatography (AKTA Purifier10 using Sephadex 75 10/300 GL [GE Healthcare, Buckinghamshire, UK]; $10 \mathrm{mM}$ Tris, $100 \mathrm{mM} \mathrm{NaCl}, \mathrm{pH} 8.0$ [running buffer] and/or PD-10 desalting columns [GE Healthcare, Piscataway, NJ]) were used to remove any unincorporated fluorophores. Fractions with an estimated fluorophoreto-protein ratio of 1.0 \pm 0.2 were pooled and used for adsorption studies.

The labeled protein was diluted to $500 \mathrm{pM}$ in binding buffer (10 mM Tris, $\mathrm{pH}$ 8.0) with added salt concentrations ranging from $1-1000 \mathrm{mM} \mathrm{NaCl}$ to vary the ionic strength. The solution was flowed over the prepared penta-argininamide modified agarose samples at 1 $\mu \mathrm{L} / \mathrm{min}$ using a Genie Plus flow system (Kent Scientific, Torrington, CT). The same region of the adsorbent was repeatedly imaged as solutions of different ionic strengths were introduced, allowing observation of the same population of individual functional adsorbent sites under differing conditions.

\subsection{Total Internal Reflectance Fluorescence (TIRF) Microscopy}

A schematic of the microscopy setup is shown in Figure 1A. The beam from a solid state $532 \mathrm{~nm}$ laser (Coherent, Compass 315M-100SL) was used for excitation and passed through an acousto-optic modulator (IntraAction, 402AF1) controlled by frequency generators (Fluke, 271-U 115V) to synchronize excitation to the detection rate and to reduce the influence of dye photophysics [41]. The beam was expanded and focused at the edge of a 1.45 NA, 100x, oil-immersion objective (Carl-Zeiss, alpha Plan-Fluar) for through-theobjective TIRF microscopy with a $1 / e$ penetration depth of $\sim 85 \mathrm{~nm}$ and incident intensity of $\sim 5 \mathrm{~mW} / \mathrm{cm}^{2}$. Emission was collected back through the objective and was separated from excitation with a notch filter (Kaiser, HNPF-532.0-1.0) and a bandpass filter (ET585/65m). An electron-multiplied charge coupled device (Andor, iXon 897) was used for detection at an integration time of $30 \mathrm{~ms}$, frame rate of $16 \mathrm{~Hz}$, and electron multiplying gain of 300 .

\subsection{Super-resolution imaging by mbPAINT}

Using TIRF microscopy, the small, fluorescent a-lactalbumin molecules can be selectively observed only when adsorbed to the interface, and are made unobservable by motion blur when freely diffusing even near the interface due to their rapid diffusion $\left(D \sim 150 \mu \mathrm{m}^{2} / \mathrm{s}\right.$ [26]) in bulk solution compared to the detector temporal resolution (16 Hz) (Figure 1B). Motion blur and the stochastic, low-density reversible adsorption of the protein to the ligands allows for super-resolution imaging in a technique we have termed motion-blur points accumulation for imaging in nanoscale topography (mbPAINT) [42].

Collected data is converted to a MATLAB (R2011b) compatible format as a series of frames. Each adsorbed protein molecule appears as a diffraction-limited point spread 
function in a single frame (Figure 1C). Pixels with intensity above a threshold three times the standard deviation of a Gaussian fit to the intensity of all pixels within the frame are identified as having an adsorbed protein present. This provides $99.7 \%$ confidence that the pixel is above the background [43] because the majority of each image is background noise, as ensured by using a low concentration of a-lactalbumin. Selected pixels are fit to a twodimensional Gaussian and the centroid location is recorded (Figure 1C). The collective centroid locations of repeated adsorption and desorption of different proteins (Figure 1D) reveal the presence of specific functional adsorption sites where penta-argininamide ligands are present, as contrasted to the bare agarose surface [21]. We then renormalize the images to distinguish events persisting over multiple frames at the same location, from events occurring in only a single frame. Expanded details of the data analysis methods can be found in Kisley et al. [21].

\subsection{Circular dichroism measurements}

Protein structural stability under different ionic strength conditions was confirmed by circular dichroism spectroscopy. A Jasco J-815 spectropolarimeter at a $1 \mathrm{~cm}$ path length, $320-250 \mathrm{~nm}, 5$ accumulations, $20^{\circ} \mathrm{C}$, and $0.1 \mathrm{~nm}$ pitch was used. For measurement, alactalbumin was diluted to $1 \mathrm{mg} / \mathrm{mL}$ in $10 \mathrm{mM}$ Tris buffer, $\mathrm{pH} 8$ at the reported $\mathrm{NaCl}$ concentrations.

\subsection{Agarose swelling measurements}

For preparation of $2 \mathrm{wt} \%$ agarose microbeads, a solution of $1 \mathrm{~g}$ of agarose (Type I-B, low EEO) in $50 \mathrm{~mL}$ of deionized water was heated to $86^{\circ} \mathrm{C}$ while stirring. A suspending solution, consisting of $9 \%$ Span- 85 in heptane was heated to $58{ }^{\circ} \mathrm{C}$ with stirring. The agarose solution was poured into the suspending solution of Span-85 and continuously stirred at $620 \mathrm{rpm}$ with an overhead stirrer. The solution was then allowed to cool and beads were washed with a $50 \%$ ethanol-water solution. Beads with radii of 140-150 $\mu \mathrm{m}$ were selected using sieves and stored in $20 \%$ ethanol-water solution at $4{ }^{\circ} \mathrm{C}$. The agarose beads were then placed in the buffer solutions with respective ionic strengths for $2 \mathrm{~h}$. The beads were placed on a coverslip and imaged on an AMG Evos XL Core light microscope. The resulting images were analyzed with ImageJ $(\mathrm{NIH}$, version $1.46 \mathrm{r})$ to extract the radius.

\subsection{Single protein adsorption kinetics}

Desorption times, defined to be the dwell time from when an immobile, adsorbed protein was first observed until it was no longer seen, were extracted as an ensemble across the entire region imaged. Individual adsorption sites were identified based on correlation analysis to find locations that had more than five protein adsorption events over 1,000 frames, a number of events statistically significantly different from the bare agarose support [21]. Desorption times are reported as cumulative distributions (integrated histograms) for higher sensitivity to rare events [44]. When comparing kinetics of the same region under different ionic strengths, image registration (MATLAB control point registration functions with an 'affine' transformation) was used to account for sample drift and to align the images. The registration was performed based on the specific adsorption sites observed in the image as control points. 


\subsection{Simulated elution profiles}

From the acquired single molecule desorption distribution, the method originally presented by Pasti et al. [45] was used to extract simulated elution curves from the single molecule desorption distributions. An adsorption-desorption equilibrium can be used to describe our system:

$$
\alpha L A+I \underset{k_{d}}{\stackrel{k_{a}}{\longrightarrow}}[\alpha L A-I]
$$

where aLA is the protein, I is the interfacial adsorption site, and [aLA-I] is the protein adsorbed to the specific adsorption site. In the stochastic theory of chromatography proposed originally by Giddings and Eyring [46] a Poisson distribution gives the probability of a single protein associating with an adsorption site $r_{m}$ times ( $r$ number of stochastic adsorption events for a given time, $t$, in the mobile phase, $m$ ):

$$
\psi_{m}=\frac{\left(k_{a} t_{m}\right)^{r_{m}}}{r_{m} !} \exp \left[-k_{a} t_{m}\right]
$$

where $k_{a}$ is the adsorption rate constant and $t_{m}$ is the total time the analyte is in the mobile phase. The Poisson distribution is converted to the frequency domain to present the expression in the characteristic function formalism $(\phi)$; this is a common method for representing stochastic processes [47]:

$$
\phi\left(t_{s} ; \omega \mid t_{m}\right)=\exp \left[\mu t_{m}\left(\exp \left\{i \omega \tau_{s}\right\}-1\right)\right] .
$$

In Equation $3, t_{s}$ is the overall time spent in the stationary phase for the analyte, $\tau_{s}$ is the duration of each individual adsorption event, and $\mu$ is the frequency of adsorption events. The expression is further modified by applying the Lévy formalism because the single molecule distribution of $\tau_{s}$ is discontinuous [48]:

$$
\phi\left(t_{s} ; \omega \mid t_{m}\right)=\exp \left[r_{m} \sum_{i=1}^{i=k}\left(\exp \left\{i \omega \tau_{s, i}\right\}-1\right) \Delta F\left(\tau_{s, i}\right)\right]
$$

where $k$ is the index of the discrete set of desorption times given by $\Delta F\left(\tau_{s}, i\right)$ that are observed in the single molecule experimental desorption time distributions. An inverse Fourier transform of the Lévy representation of the characteristic function to the time domain is then used to extract $f\left(t_{s}\right)$, the simulated chromatographic peak:

$$
\phi\left(t_{s} ; \omega \mid t_{m}\right) \stackrel{F T}{\longrightarrow} f\left(t_{s}\right) .
$$

The stochastic chromatographic theory was developed for columns, but the single molecule experiments do not involve a defined column residence time, and so $r_{m}$ in Equation 4 is not defined. Instead, a set value of $r_{m}=100$ was estimated based on experimental conditions, including observation volume, flow rate, protein concentration, and average number of detected events over time and was used to simulate relative elution curves for comparisons unique to the single molecule imaging conditions employed. 


\section{Results and Discussion}

\subsection{Super-resolution imaging shows narrowing of the functional adsorbent site population at high ionic strength}

Super-resolution event analysis (Figure 2) demonstrates that, as the solution ionic strength is increased, a-lactalbumin adsorption occurs at fewer functional adsorption sites on the substrate. mbPAINT super-resolution images of a-lactalbumin adsorption on the same area of the clustered-charge penta-argininamide functionalized agarose stationary phase at ionic strengths ranging from $1 \mathrm{mM}$ to $1000 \mathrm{mM}$ are shown in Figure 2A-D (images of larger regions of interest are included in supplementary material, Figure S1). As can be seen in the images, and as quantified by the event analysis in Figure 2E, at salt concentrations higher than $1 \mathrm{mM} \mathrm{NaCl}$, there are fewer active adsorption sites. This result is consistent with ionexchange elution by salt, as well as ensemble measurements of decreased protein adsorption at increasing salt concentration due to electrostatic shielding [5-15,22,49,50].

Further analysis of the data in Figure 2 suggests, however, that the mechanism of saltdependence on separations efficiency is driven by more than just the expected shift in the electrostatic shielding equilibrium. Although the number of active sites narrows with increasing salt (Figure 2E), the number of adsorption events per participating site increases (Figure 2F). There are several possible explanations for the unexpected relationship between number of active sites and adsorption events per site, including salt-induced protein denaturation, salt-induced agarose structural changes, and complex adsorption/desorption kinetics. As discussed below, the additional level of detail provided by single-molecule event analysis allows us to examine detailed kinetics, while structural analyses are used to examine protein and support conformation.

\subsection{Circular dichroism spectroscopy shows ionic strength does not significantly change the structure of $a$-lactalbumin}

To test for any influence of varying salt concentration on protein structure, the $\mathrm{CD}$ spectrum of a-lactalbumin was measured in buffers of ionic strength varying over the range of interest in this work (Figure 3). The CD spectra in Figure 3 clearly show that there is no significant alternation of protein conformation at any of the $\mathrm{NaCl}$ buffer conditions that were employed, as the structure-sensitive region near $270 \mathrm{~nm}$ [51] remains unchanged. Thus, we can conclude that the event statistics shown in Figure 2 are not significantly attributable to protein structural effects of salt.

\subsection{Decreased swelling of agarose at increased ionic strength could cause change in steric availability of ligands}

Ion-exchange adsorbents can undergo structural changes with changes in ionic strength as inter-charge electrostatic repulsion is more or less well screened [52-55]. There also can be direct effects of salt on the structure of agarose itself [56]. Instead of indirect measurements on the single-molecule agarose film, the swelling of model low-charge agarose microbeads as a function of ionic strength was examined directly via optical imaging (Figure 4). Figure 4 shows the size distribution of agarose microbeads as a function of ionic strength, and the radius extracted via an error function fit. Microbead analysis was chosen because it is 
difficult to directly test the agarose film for salt-induced structural changes; however it is known that such relationships do exist [56], and the analysis chosen here is one way to quantify the relationship. Indeed, a decrease in the size of the microbeads is observed as ionic strength increases. This data shows that the swelling of the agarose decreases at higher ion concentrations, which in turn would cause a reduction in pore size within the agarose support. It is important to note that the preparation and chemistry of the agarose microbeads is not identical to that of the agarose thin films used in the single-molecule adsorption experiments (see Materials and Methods for details), and that local charge density at adsorbent sites is relatively high. However, that the bead data exhibits such strong swelling/ contraction under the same wide range of salt conditions used for the adsorption analysis strongly supports the notion that steric accessibility within the support does play a role in the observed site-specific statistical behavior shown in Figure 2, and the high local charge density at functional adsorption sites would only increase the potential role of salt-dependent changes in agarose structure around these sites.

\subsection{Desorption kinetic analysis relates ensemble adsorption behavior and super-resolution event statistics}

Single molecule a-lactalbumin desorption kinetics at multiple penta-argininamide adsorption sites were extracted and presented in Figure 5 as cumulative decay curves as a function of $\mathrm{NaCl}$ concentration. A qualitative analysis of the ionic strength dependent decays suggests already that the kinetics are complicated, as the curves are clearly multiexponential. This is consistent with our previous observation of inter-site heterogeneity, and reflects that multiple processes are contributing to the observed adsorption statistics [21]. Quantitative analysis was achieved by fitting to either a two- or three-component exponential decay with standard curve fitting considerations [57] to account for the heterogeneity present among sites [21]. The resulting fitted decay times, their amplitudes and uncertainties, and the weighted average decay times, are included in Table 1 . The first result of kinetic analysis is that, when the multi-exponential time and amplitude components are combined to yield a weighted ensemble average lifetime, the trend in desorption kinetics follows very clearly the overall trend observed in chromatography and in ensemble experiments: elution is faster at higher salt because the majority of adsorption times are smaller.

More interestingly, however, the single-molecule analysis allows a detailed comparison of how the multiple kinetic components change as a function of ionic strength. The fastest component, on the order of tens of milliseconds, is least affected by ionic strength, and likely reflects a convolution of our analysis resolution $(3 \mathrm{~ms})$ and interfacial protein diffusion, which has been observed to occur on these time scales $[26,58,59]$. An intermediate component on the order of 100-200 ms also is observed; its overall lifetime and contribution to the overall decay decrease as the ionic strength is increased. This component, which follows the expected electrostatic shielding trend, most likely reflects the saltmediated Coulombic interaction between protein and peptide. Finally, there is a longer component of several hundred milliseconds, for all but the lowest ionic strength. As this component is not observed at the lowest salt condition, increases in duration as ionic strength increases, and follows the trend of contracting agarose described in Figure 4, we 
propose to attribute this component to steric trapping at active adsorbent sites within contracted agarose pores. Overall, the result of decreased low affinity interactions at increased ionic strengths agrees with our previous ensemble-based observations of saltmediated shielding [12,60], but offer the additional information that ionic-strength induced steric effects also contribute, reflected in both the multi-exponential kinetics and the ensemble averaged kinetics. Tuning both of these behaviors, and perhaps also the interfacial diffusion component that was unaffected by ionic strength, should allow more-detailed control of elution times and distributions.

\subsection{Increased ionic strength reduces heterogeneity effects on protein elution curves}

There is a substantial body of ensemble adsorption isotherm studies that support the idea that protein ion-exchange adsorption displays an inherent heterogeneity [6-11,45,46], with affinity decreasing with adsorbate loading (apparent "negative cooperativity"). While interadsorbate repulsion and adsorbate heterogeneity can contribute to such behavior, systems in which these factors are avoided still show heterogeneity, which we and others ascribe to variations in the properties of the adsorbent itself due to steric and structural variations in the support, and stochastic clustering of charged ligands in conventional adsorbents produced by random chemical derivatization with monovalent charged ligands .

In addition, we have repeatedly observed a decrease in the apparent adsorption heterogeneity in the presence of conditions which make adsorption more difficult, e.g. increased ionic strength or a presence of a competitor protein. Specific examples from our related prior work include the adsorption of cytochrome $\mathrm{c}$ and its charge mutant forms on Mono $\mathrm{Q}$ beads, where the Hill cooperative factor $n_{\mathrm{H}}$ decreased with increasing salt concentration [60] and the binding of a-lactalbumin on clustered charge penta-argininamide adsorbents [12] where the adsorbents generally exhibited negative cooperativity with a value of $n_{\mathrm{H}}$ less than one. On increasing the salt concentration, the value of $n_{\mathrm{H}}$ shifted closer to unity, indicating a lower degree of heterogeneity, and suggesting the participation of a reduced set of more homogeneous functional adsorbent sites. Moreover, in what we believe were the first true competitive protein adsorption isotherms (in the strict sense of "isotherm", with all intensive variables held constant including competitor concentration), at higher competitor concentrations the heterogeneity of the anion-exchange adsorption of a test protein was decreased [7]. We believe this reflects the existence of a heterogeneous population of sites with different affinities, on which different proteins compete with varying success. Each of these examples supports our speculation that, in the presence of adsorption-suppressing conditions, weak adsorption on lower affinity sites is suppressed; only a more uniform population of sites is left to participate in binding and functional heterogeneity is reduced.

Although the cost of penta-argininamide adsorbent makes it cost prohibitive to perform multiple column separations to experimentally test the above hypotheses, it is possible to simulate isocratic elution profiles from the kinetic curves (Figure 6) by combining the ensemble desorption time kinetics (Figure 5), and the stochastic theory of ion-exchange chromatography (Eqs. 4-5 above). Indeed, the simulated elution data (Figure 6) show that the narrowest and fastest elution curve was obtained at the highest ionic strength of $1 \mathrm{M}$ $\mathrm{NaCl}$. It is noteworthy that the desorption data are used directly as the major input to these 
simulations, and the simulations do not depend upon any fitting of the complex desorption kinetics curves or extraction of major kinetic components. The similarity of the 1 and 10 $\mathrm{mM} \mathrm{NaCl}$ results likely reflects the relatively large influence of the $5 \mathrm{mM}$ ionic strength contribution of the buffer (10 mM Tris $\mathrm{HCl}, \mathrm{pH} 8.0)$ at the low salt levels. For comparison, the supplementary material includes the elution profile simulated at infinite salt concentration (interactions with agarose support only, Figure S3).

The participation of a narrower and more homogeneous population of functional adsorption sites at high ionic strength is evident in the chromatographic elution curves predicted by the stochastic model. Isocratic retention at higher salt concentrations produces sharper peaks, with a HETP (height equivalent to a theoretical plate; Figure 6, inset) three-fold smaller at higher ionic strength. While the statistical model and the thin film adsorbent do not fully capture important transport aspects of real chromatographic systems, this result is consistent with our and others' reports of lower apparent heterogeneity of adsorption isotherms at higher salt concentration.

The results presented above strongly support the role of the spatial variations in adsorbent structural/energetic properties in the observed heterogeneity of protein ion-exchange adsorption isotherms, and provide the first direct observation of the mechanism of the narrowing of the functional site population as ionic strength increases. Moreover, these results are consistent with previous single-molecule based findings by our group, where variations in the agarose support led to heterogeneity in desorption times among chemicallyidentical clustered-charge ligands [21] and a decrease in solute mobility near, on, and inside the porous substrate [26]. Some differences between our systems and systems based on porous particles and packed columns should be noted. First, the sample is a thin-film interface, $\sim 80 \mathrm{~nm}$ thick. Using TIRF microscopy, our measurements are limited to $\sim 100 \mathrm{~nm}$ from the glass-agarose interface. Therefore, the measurements are representative of the mobile phase-stationary phase interface and the first $80 \mathrm{~nm}$ of the agarose support, as compared to practical column packing materials tens to hundreds of microns in size. Also, the loading of the ligand, here an intentionally clustered-charge peptide, penta-argininamide, is deliberately kept very low to form resolvable, non-interacting adsorption sites. In conventional practice, ligands are loaded at levels three to four orders of magnitude higher, leading to protein adsorption at stochastic clusters of ligands with a heterogeneous distribution, where this "narrowing" phenomenon likely is even more pronounced.

\section{Conclusions}

Direct single-molecule observation of the behavior of single functional adsorption sites on a realistic ion-exchange agarose adsorbent shows that the population of functional sites able to participate in protein adsorption is narrowed at higher ionic strength. Structural analyses of the protein and support, and desorption kinetic analyses, suggest that this narrowing of the active adsorbent site population is due both to electrostatic screening between the protein and ligand, and the tuning of the steric availability within the agarose support. The ensemble-averaged results are consistent with the observed heterogeneity of protein ionexchange adsorption at single-molecule level, and the heterogeneity reduction induced by 
conditions which make adsorption more difficult, such as increased salt concentration and presence of competitor proteins.

\section{Supplementary Material}

Refer to Web version on PubMed Central for supplementary material.

\section{Acknowledgments}

C.F.L. thanks the NSF [CBET-1134417] and [CHE-1151647]; the Welch Foundation [C-1787], and the NIH [GM94246-01A1], for support of this work. R.C.W. thanks the NSF [CBET-1133965] and the Welch Foundation [E-1264]. L. K. thanks the NSF for Graduate Research Fellowship 0940902. We thank S. Link and research group for discussions.

\section{References}

1. Bowen WR, Hughes DT. J. Colloid Interf. Sci. 1993; 158:395.

2. Dismer F, Hubbuch J. J. Chromatogr. A. 2007; 1149:312. [PubMed: 17439816]

3. Hart DS, Harinarayan C, Malmquist G, Axén A, Sharma M, van Reis R. J. Chromatogr. A. 2009; 1216:4372. [PubMed: 19168177]

4. Roth CM, Unger KK, Lenhoff AM. J. Chromatogr. A. 1996; 726:45.

5. Lan Q, Bassi AS, Zhu J-XJ. A. Margaritis, Chem. Eng. J. 2001; 81:179.

6. Asthagiri D, Lenhoff AM. Langmuir. 1997; 13:6761.

7. Cano T, Offringa ND, Willson RC. J. Chromatogr. A. 2005; 1079:116. [PubMed: 16038297]

8. Chang C, Lenhoff AM. J. Chromatogr. A. 1998; 827:281. [PubMed: 9914656]

9. Johnson RD, Arnold FH. Biotech. Bioeng. 1995; 48:437.

10. Johnson RD, Arnold FH. Biochim. Biophys. Acta (BBA)-Nucleic Acids and Protein Synthesis. 1995; 1247:293.

11. Skidmore GL, Chase HA. J. Chromatogr. 1990; 505:329. [PubMed: 2355064]

12. Fu JY, Balan S, Potty A, Nguyen V, Willson RC. Anal. Chem. 2007; 79:9060. [PubMed: 17973458]

13. Gill DS, Roush DJ, Willson RC. J. Colloid Interf. Sci. 1994; 1:1.

14. Brooks CA, Cramer SM. AIChE J. 1992; 38:1969.

15. Yamamoto S, Yoshimoto N, Nishizumi Y. J. Chromatogr. A. 2009; 1216:2612. [PubMed: 19211103]

16. Yao Y, Lenhoff AM. Anal. Chem. 2005; 77:2157. [PubMed: 15801750]

17. Ortego BC, Whittenton JJ, Li H, Tu SC, Willson RC. Biochemistry. 2007; 46:13864. [PubMed: 17985929]

18. Yao Y, Czymmek KJ, Pazhianur R, Lenhoff AM. Langmuir. 2006; 22:11148. [PubMed: 17154596]

19. Yao Y, Lenhoff AM. J. Chromatogr. A. 2006; 1126:107. [PubMed: 16844131]

20. Johnson RD, Arnold FH. Biotechnol. Bioeng. 1995; 48:437. [PubMed: 18623507]

21. Kisley L, Chen J, Mansur AP, Shuang B, Kourentzi K, Poongavanam MV, Chen WH, Dhamane S, Willson RC, Landes CF. Proc Natl Acad Sci U S A. 2014 DOI: 10.1073/pnas.1318405111.

22. Kroner F, Hubbuch J. J. Chromatogr. A. 2013; 1285:78. [PubMed: 23489486]

23. Stone MC, Carta G. J. Chromatogr. A. 2007; 1160:206. [PubMed: 17560582]

24. Dziennik SR, Belcher EB, Barker GA, Lenhoff AM. Biotech. Bioeng. 2005; 91:139.

25. Xiao Y, Stone T, Bell D, Gillespie C, Portoles M. Anal. Chem. 2012; 84:7367. [PubMed: 22803776]

26. Daniels CR, Kisley L, Kim H, Chen W-H, Poongavanam M-V, Reznik C, Kourentzi K, Willson RC, Landes CF. J. Mol. Recognit. 2012; 25:435. [PubMed: 22811068] 
27. Cuppett CM, Doneski LJ, Wirth MJ. Langmuir. 2000; 16:7279.

28. Ludes MD, Wirth MJ. Anal. Chem. 2001; 74:386. [PubMed: 11811413]

29. Wirth MJ, Legg MA. Annu. Rev. Phys. Chem. 2007; 58:489. [PubMed: 17105411]

30. Wirth MJ, Ludes MD, Swinton DJ. Anal. Chem. 1999; 71:3911.

31. Wirth MJ, Swinton DJ. Anal. Chem. 1998; 70:5264.

32. Wirth MJ, Swinton DJ, Ludes MD. J. Phys. Chem. B. 2003; 107:6258.

33. Isailovic S, Li H-W, Yeung ES. J. Chromatogr. A. 2007; 1150:259. [PubMed: 17054967]

34. Kang SH, Shortreed MR, Yeung ES. Anal. Chem. 2001; 73:1091. [PubMed: 11305636]

35. Kang SH, Yeung ES. Anal. Chem. 2002; 74:6334. [PubMed: 12510756]

36. Kwok KC, Yeung KM, Cheung NH. Langmuir. 2007; 23:1948. [PubMed: 17279679]

37. Cooper JT, Peterson EM, Harris JM. Anal. Chem. 2013; 85:9363. [PubMed: 23998479]

38. Chen, W.-h.; Fu, JY.; Kourentzi, K.; Willson, RC. J. Chromatogr. A. 2011; 1218:258. [PubMed: 21159348]

39. Afanassiev V, Hanemann V, Wölfl S. Nucleic Acids Res. 2000; 28:e66. [PubMed: 10871389]

40. Xavier A, K. Willson RC. Biophys. J. 1998; 74:2036. [PubMed: 9545062]

41. Kisley L, Chang W-S, Cooper D, Mansur AP, Landes CF. Methods Appl. Fluoresc. 2013; 1:037001. [PubMed: 24587894]

42. Chen J, Bremauntz A, Kisley L, Shuang B, Landes CF. ACS Appl. Mater. Interf. 2013; 5:9338.

43. Peterson EM, Harris JM. Anal. Chem. 2009; 82:189. [PubMed: 19957961]

44. Walder R, Kastantin M, Schwartz DK. Analyst. 2012; 137:2987. [PubMed: 22617120]

45. Pasti L, Cavazzini A, Felinger A, Martin M, Dondi F. Anal. Chem. 2005; 77:2524. [PubMed: 15828789]

46. Giddings JC, Eyring H. J. Phys. Chem. 1955; 59:416.

47. Dondi F, Remelli M. J. Phys. Chem. 1986; 90:1885.

48. Dondi F, Cavazzini A, Pasti L. J. Chromatogr. A. 2006; 1126:257. [PubMed: 16815427]

49. Kopaciewicz W, Regnier F. Anal. Biochem. 1983; 133:251. [PubMed: 6638483]

50. Stone MC, Tao Y, Carta G. J. Chromatogr. A. 2009; 1216:4465. [PubMed: 19342054]

51. Griko YV, Remeta DP. Protein Sci. 1999; 8:554. [PubMed: 10091658]

52. Boyde TRC. J. Chromatogr. 1976; 124:219.

53. Janson, J-C.; Jönsson, JÄ. Introduction to Chromatography. John Wiley \& Sons, Inc.; 2011.

54. Strobel, G-J. Ion Exchangers. Dextran and Agarose Ion Exchangers. Walter de Gruyter \& Co.; Berlin: 1990.

55. Livney YD, Ramon O, Kesselman E, Cogan U, Mizrahi S, Cohen Y. Journal of Polymer Science Part B: Polymer Phys. 2001; 39:2740.

56. Piculell L, Nilsson S. J. Phys. Chem. 1989; 93:5596.

57. Zielesny, A. From Curve Fitting to Machine Learning: An Illustrative Guide to Scientific Data Analysis and Computational Intelligence. Springer; Berlin: 2011.

58. Reznik C, Estillore N, Advincula RC, Landes CF. J. Phys. Chem. B. 2009; 113:14611. [PubMed: 19813742]

59. Reznik C, Landes CF. Acc. Chem. Res. 2012; 45:1927. [PubMed: 23013103]

60. Gill DS, Roush DJ, Willson RC. J. Chromatogr. A. 1994; 684:55. [PubMed: 7987477] 


\section{Highlights}

- Direct observation of individual functional ion-exchange ligands at varying salt.

- Elution curves were assembled by combining ensemble kinetics and stochastic theory.

- Ionic strength reduces heterogeneity of active adsorption sites.

- Electrostatic screening and steric availability within the agarose support play a role.

- Results help interpret a large body of previous results. 


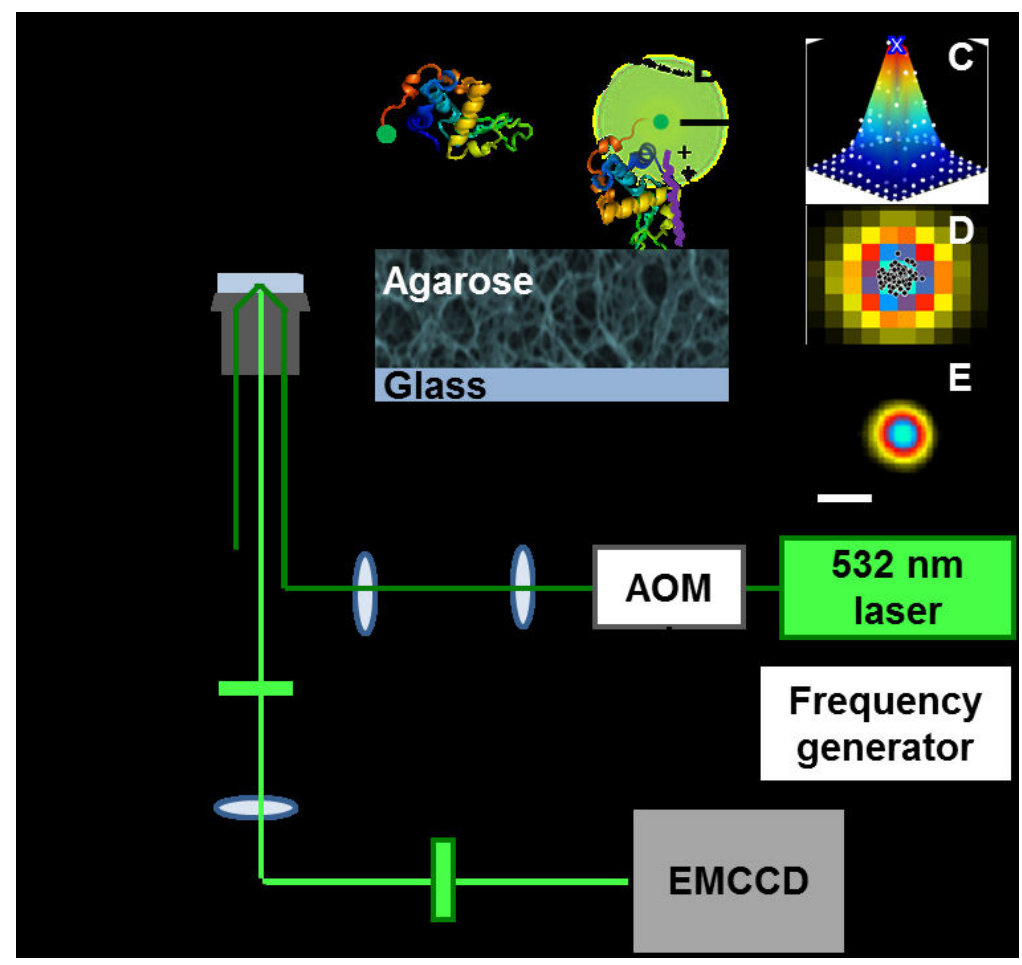

Figure 1.

Instrument, experiment, and mbPAINT analysis. (A) Total internal reflectance fluorescence microscope used to collect data. An acousto-optic modulator (AOM) and frequency generator are used to synchronize excitation and detection rates to prevent photophysical effects from interfering with measurements [41]. (B) Cartoon representation of alactalbumin protein at the penta-argininamide functionalized agarose stationary phase. The emission from the fluorescent label on the protein is only observable when it is adsorbed to the interface, not when it is freely diffusing in the bulk, allowing for (C-E) mbPAINT analysis [42]. (C) The data from the point spread function emission (white dots) is fit to a 2D Gaussian (surface) to obtain the centroid location (blue $\mathrm{x}$ ). (D) Centroids from $\sim 100$ adsorption events (overlaid the diffraction limited image) localized the ligand to $\sim 30 \mathrm{~nm}$, shown in $(E)$, the renormalized pseudo super-resolution image. Scale bar $=200 \mathrm{~nm}$. 


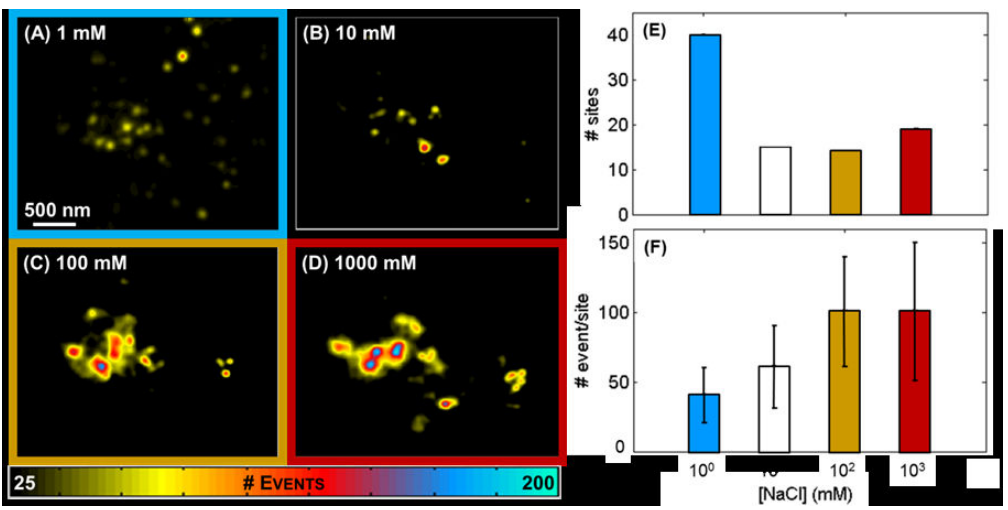

Figure 2.

Adsorption of a-lactalbumin at the penta-argininamide/agarose interface under different ionic strength conditions. (A, B, C, D) Super-resolution pseudo images of the same region of the sample obtained from 5,000 frames of data in $10 \mathrm{mM}$ Tris, $\mathrm{pH} 8.0$ with added 1, 10, 100, $1000 \mathrm{mM} \mathrm{NaCl}$, respectively. Larger area images are presented in the SI. Quantified number of $(\mathrm{E})$ specific binding sites and $(\mathrm{F})$ events per site from the super-resolution images. 


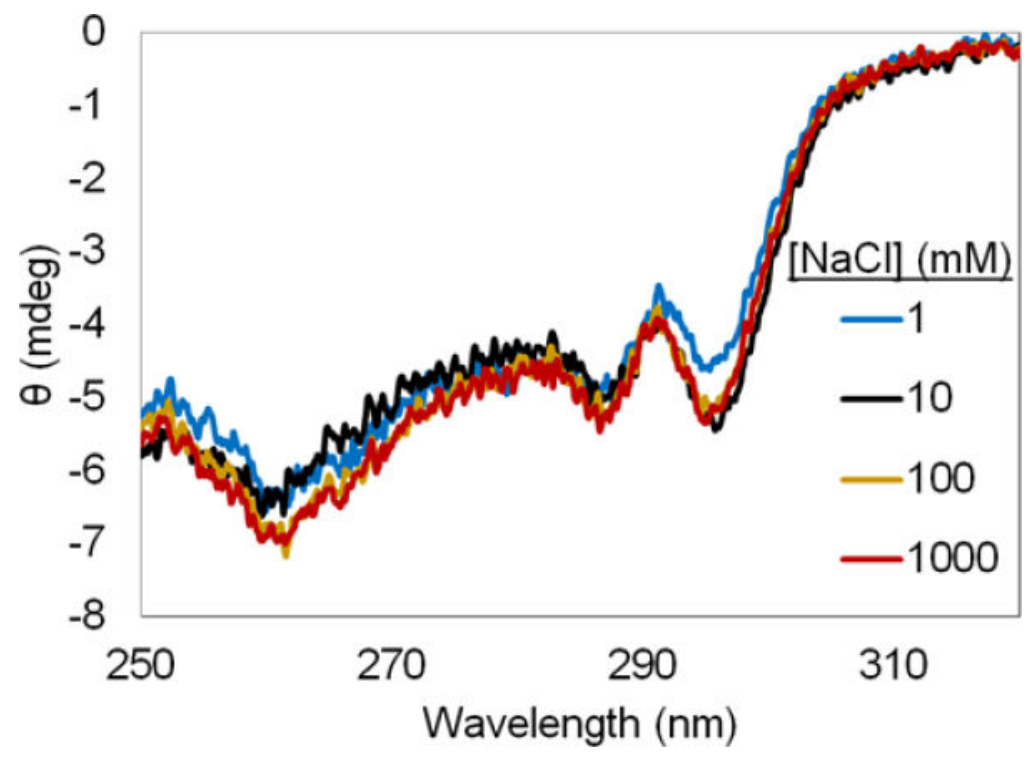

Figure 3.

Circular dichroism spectra of a-lactalbumin in $10 \mathrm{mM}$ Tris, $\mathrm{pH} 8.0$ with added 1, 10, 100, or $1000 \mathrm{mM} \mathrm{NaCl}$, showing the weak influence of salt concentration on the protein's structure. 


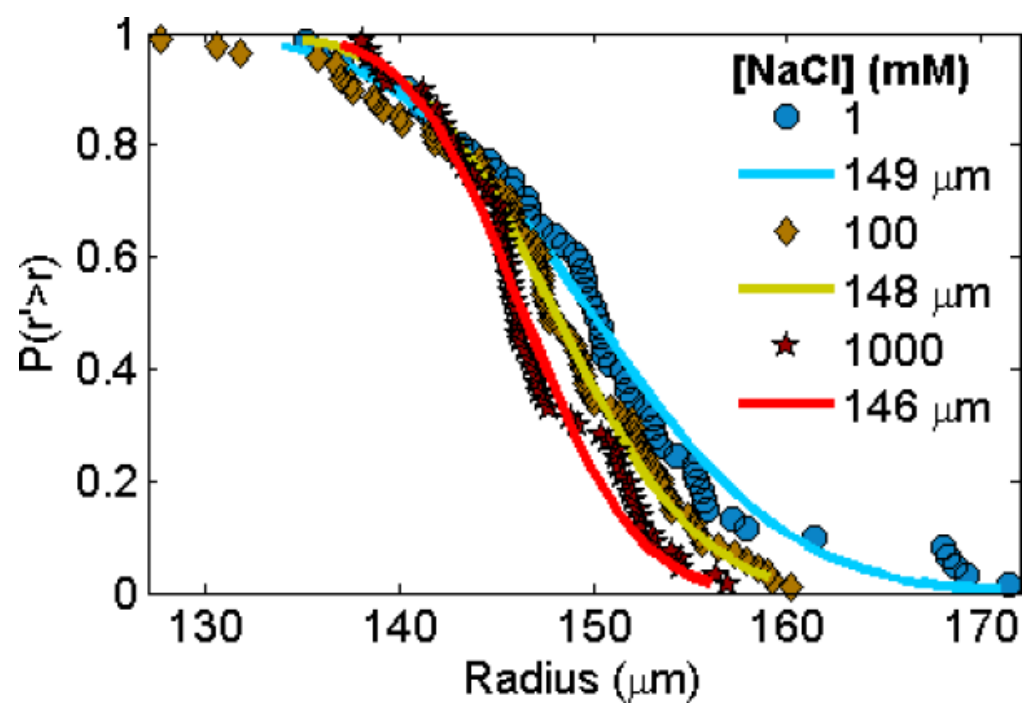

Figure 4.

Radii of agarose microbeads as a function of ionic strength. Cumulative distribution of the radius of agarose microbeads extracted from light microscopy images. Average radius extracted from error function fits to the data (solid lines) are listed in legend. $(n=61,88,67$ for $1,100,1000 \mathrm{mM}$, respectively) 


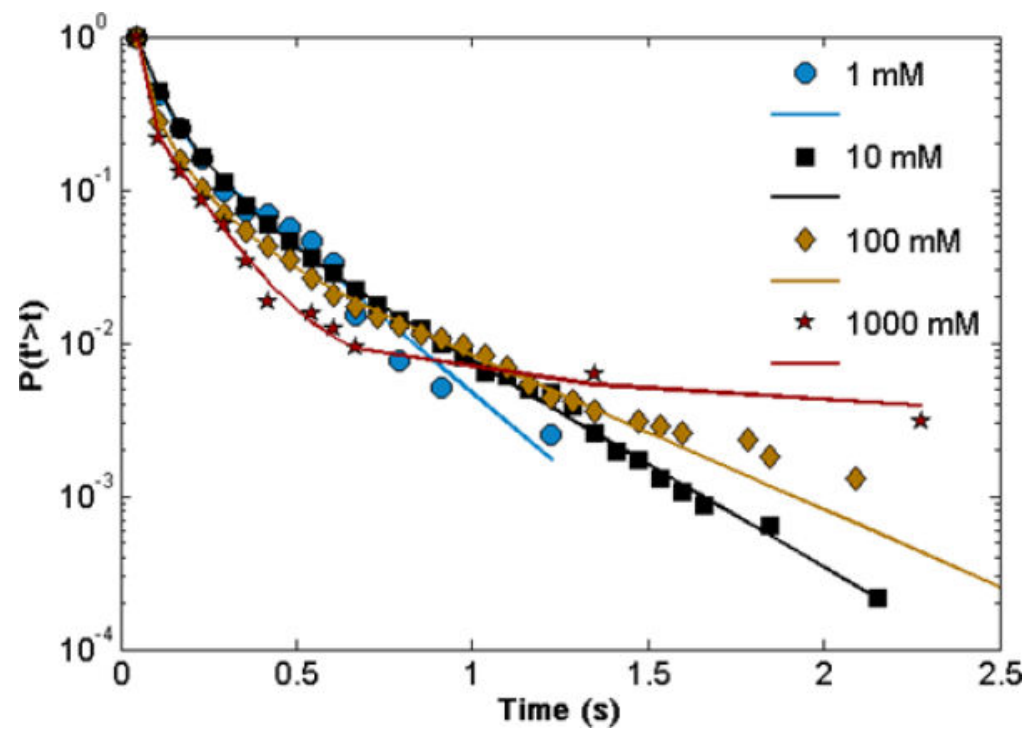

Figure 5.

Cumulative distributions of desorption times obtained from ensemble of $>100$ individual penta-argininamide ligands in $10 \mathrm{mM}$ Tris, pH 8.0 with $1 \mathrm{mM}-1000 \mathrm{mM} \mathrm{NaCl}$ (nonlinear least squared fits shown by solid lines). Details of fit parameters and dual-linear plots of these data are presented in Table 1 and the SI. 


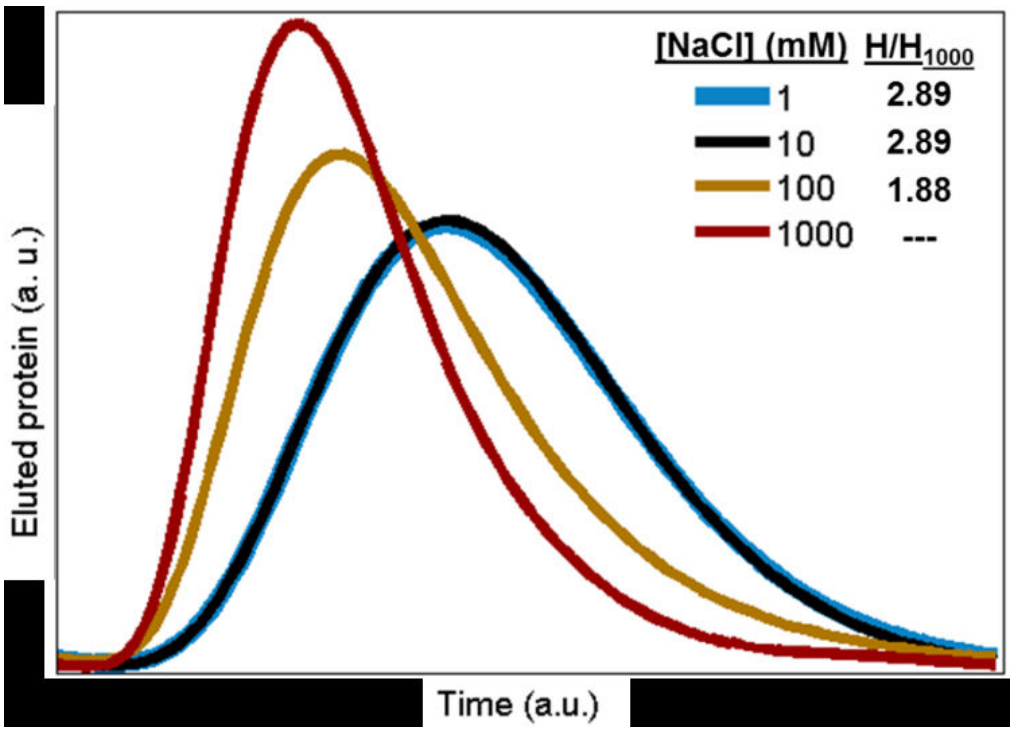

Figure 6.

Simulated elution curves obtained from statistical simulations of the ensemble analysis of desorption times of a-lactalbumin at penta-argininamide ligands under different ionic strengths. (inset) Relative efficiencies as measured by plate height (HETP) at different ionic strengths compared to best-performing $1000 \mathrm{mM}$ conditions. 


\section{Table 1}

Ensemble kinetics fitting results

\begin{tabular}{|c|c|c|c|c|}
\hline$[\mathbf{N a C I}](\mathbf{m M})$ & 1 & 10 & 100 & 1000 \\
\hline Averaged $\tau$ (s) & 0.080 & 0.074 & 0.040 & 0.029 \\
\hline Amplitude Fast & $81 \pm 4 \%$ & $63 \pm 1 \%$ & $89 \pm 6 \%$ & $90 \pm 80 \%$ \\
\hline$\tau 1(s)$ & $0.047 \pm 0.005$ & $0.034 \pm 0.002$ & $0.024 \pm 0.001$ & $0.014 \pm 0.004$ \\
\hline Amplitude Slow 1 & $19 \pm 3 \%$ & $30 \pm 2 \%$ & $9.7 \pm 0.8 \%$ & $2.6 \pm 0.2 \%$ \\
\hline$\tau 2(s)$ & $0.22 \pm 0.02$ & $0.104 \pm 0.006$ & $0.107 \pm 0.008$ & $0.13 \pm 0.01$ \\
\hline Amplitude Slow 2 & - & $7.0 \pm 0.8 \%$ & $1.6 \pm 0.2 \%$ & $0.4 \pm 0.3 \%$ \\
\hline$\tau 3(s)$ & - & $0.32 \pm 0.01$ & $0.43 \pm 0.03$ & $3.0 \pm 5.5$ \\
\hline
\end{tabular}

